\title{
Assessment of Terra MODIS thermal emissive band calibration using cold targets and measurements in lunar roll events
}

Tiejun Chang, Xiaoxiong Xiong, Ashish Shrestha, Aisheng $\mathrm{Wu}, \mathrm{Na}$ Chen, et al.

Tiejun Chang, Xiaoxiong Xiong, Ashish Shrestha, Aisheng Wu, Na Chen, Yonghong Li, Qiaozhen Mu, "Assessment of Terra MODIS thermal emissive band calibration using cold targets and measurements in lunar roll events," Proc. SPIE 10785, Sensors, Systems, and Next-Generation Satellites XXII, 107851C (25 September 2018); doi: 10.1117/12.2325378

SPIE. Event: SPIE Remote Sensing, 2018, Berlin, Germany 


\title{
Assessment of Terra MODIS Thermal emissive band calibration using cold targets and measurements in lunar roll events
}

\author{
Tiejun Chang *a Xiaoxiong (Jack) Xiong ${ }^{\mathrm{b}}$, Ashish Shrestha ${ }^{\mathrm{a}}$, Aisheng $\mathrm{Wu}^{\mathrm{a}}, \mathrm{Na}^{\mathrm{C}} \mathrm{Chen}^{\mathrm{a}}$, \\ Yonghong $\mathrm{Li}^{\mathrm{a}}$, and Qiaozhen $\mathrm{Mu}^{\mathrm{a}}$ \\ ${ }^{a}$ Science Systems and Applications, Inc, Lanham, MD 20706; \\ ${ }^{\mathrm{b}}$ Sciences and Exploration Directorate, NASA/GSFC, Greenbelt, MD 20771
}

\begin{abstract}
Terra MODIS has provided continuous global observations for science research and applications for more than 18 years. The MODIS Thermal emissive bands (TEB) radiometric calibration uses a quadratic function for instrument response. The calibration coefficients are updated using the response of an on-board blackbody (BB) in quarterly warm-up and cool-down (WUCD) events. As instrument degradation and electronic crosstalk of long-wave infrared (LWIR) bands 27 to 30 developed substantial issues, accurate calibration is crucial for a high-quality L1B product. The on-board BB WUCD temperature ranges from $270 \mathrm{~K}$ to $315 \mathrm{~K}$ and the derived nonlinear response has a relatively large uncertainty for the offset, especially for these LWIR bands, which affects the measurements of low brightness temperature (BT) scenes.

In this study, the TEB radiometric calibration impact on the L1B product is assessed using selected cold targets and the measurements during regular lunar rolls. The cold targets include Antarctic Dome Concordia (Dome-C) and deep convective clouds (DCC) for the calibration assessment, focusing on bands 27 to 30 . Dome-C area is covered with uniformly-distributed permanent snow, and the atmospheric effect is small and relatively constant. Usually the DCC is treated as an invariant earth target to evaluate the reflective solar band calibration. The DCC can also be treated as a stable target to assess the performance of TEB calibration. During a scheduled lunar observation event with a spacecraft roll maneuver to view the moon through the space view port, the instrument cavity provides a stable reference for calibration assessment. The long-term trending of BT measurements and the relative difference between scan mirror sides and detectors are used for the assessment of the calibration consistency and stability. The comparison of L1B products over the selected targets before and after the calibration coefficients update can be used to assess the impact of a calibration look-up table (LUT) update. This assessment is beneficial for future calibration algorithm and LUT update procedure improvements for enhancing the L1B product quality.
\end{abstract}

Keywords: MODIS, Radiometric calibration, TEB, DCC, Dome-C

\section{INTRODUCTION}

The Moderate Resolution Imaging Spectroradiometer (MODIS) onboard the Terra satellite has operated for more than 18 years in mission since its launch in 1999 [1-4]. The instrument has provided continuous global observations for science research and applications. Measurements from the MODIS instrument have produced an unprecedented amount of science data products with primary applications for studies of the Earth's land, ocean and atmosphere [5-9]. MODIS has 36 spectral bands, where bands 1 to 19 and 26 are reflective solar bands (RSB) covering a wavelength range of $0.41 \mu \mathrm{m}$ to $2.1 \mu \mathrm{m}$ and bands 20 to 25 and 27 to 36 are thermal emissive bands (TEB) with a wavelength range of $3.8 \mu \mathrm{m}$ to $14.2 \mu \mathrm{m}$. The onboard solar diffuser (SD) and Blackbody (BB), together with lunar observations and Earth view measurements, are used for post-launch calibrations. After 18 years of on-orbit operation, the electronic crosstalk among bands 27 to

Sensors, Systems, and Next-Generation Satellites XXII, edited by Steven P. Neeck, Philippe Martimort, Toshiyoshi Kimura, Proc. of SPIE Vol. 10785, 107851C · C 2018 SPIE · CCC code: 0277-786X/18/\$18 · doi: 10.1117/12.2325378 
30 has increased substantially which caused biases in the EV brightness temperature (BT) measurements, surface feature contamination, and other downstream products such as water vapor and cloud mask. The crosstalk in these bands has been characterized and corrected using lunar measurements. The calibration coefficients obtained from lunar measurements are updated regularly to maintain accuracy and consistency of the L1B product, especially for the increasing contamination from electronic cross-talk in the PV LWIR bands. This is particularly important to characterize the instrument response change after the February 2016 safe mode [10]. The electronic cross-talk correction for bands 27-30 of Terra MODIS is derived using observations of the Moon. Lunar roll event are scheduled monthly and the cross-talk coefficients are updated whenever the change in the coefficients exceed the update criterion. The correction of the PV LWIR bands cross-talk has been implemented in Collection 6.1 (C6.1) for both on-orbit calibrations and Earth radiance retrieval over the entire mission [10]. The implementation of cross-talk correction results in improvement of calibration accuracy and reduction of image artifacts.

During quarterly-scheduled warm-up and cool-down (WUCD) on-orbit calibrations, the BB temperature ranges from $270 \mathrm{~K}$ to $315 \mathrm{~K}$. The instrument nonlinear coefficients, which are derived using the WUCD data, are updated following the LUT delivery procedure $[11,12]$. Due to the challenge of accurately determining $\mathrm{a}_{0}$ from these on-orbit WUCD calibrations, the C6.1 algorithm handles a0 differently for the two mirror sides. To assess the impact of the $\mathrm{a}_{0}$ uncertainty, cold targets can be used to provide a good indication for the calibration consistency between the two mirror sides. Due to the degradation in the instrument performance after the safe mode event of Feb. 2016, it has become necessary to revisit the existing $\mathrm{a}_{0}$ and $\mathrm{a}_{2}$ coefficients update procedure, particularly for the PV LWIR bands.

This paper focuses on the analysis of the Terra MODIS TEB nonlinear calibration coefficients $\mathrm{a}_{0}$ and $\mathrm{a}_{2}$ LUT update procedure and its impact on the Level 1B (L1B) product for C6.1 [10-12]. The cold targets, Dome-C and deep convective clouds (DCC), are used for this assessment. The scheduled lunar events provide the measurement of the cavity temperature that is lower than the BB nominal temperature. Cavity measurements provide another reliable data source to assess the calibration consistency. Section 2 presents the background of the MODIS calibration algorithm for the TEB and the Terra safe mode in February 2016. The Terra calibration update and its impact on the L1B product are presented in section 3. Section 4 discusses the assessments using cold targets and scheduled lunar events.

\section{BACKGROUND}

\subsection{MODIS calibration algorithm and Collection 6.1}

MODIS has 16 TEB covering the mid-wave infrared (MWIR: bands 20-25) and long-wave infrared (LWIR: bands 27-36) spectral regions. All TEB are located on two cold focal plane assemblies (CFPAs): a short-wave and mid-wave infrared (SMIR) FPA and a long-wave infrared (LWIR) FPA. Bands 20-30 consist of ten photovoltaic (PV) detectors per band, while bands 31-36 consist of ten photoconductive (PC) detectors per band. The CFPAs are nominally controlled on-orbit at $83 \mathrm{~K}$ using a passive radiative cooler. An on-board calibrator blackbody (BB) is used for the TEB calibration, with a variant temperature ranging from instrument ambient temperature (about $270 \mathrm{~K}$ ) to $315 \mathrm{~K}$ measured using a set of 12 uniformly-distributed thermistors. Normally, it is set at $290 \mathrm{~K}$ for Terra MODIS, as shown in Figure 1. The on-board BB serves as the primary calibration source while the space view (SV) provides a reference for the instrument background and offsets. MODIS TEB calibration uses a quadratic calibration algorithm on a scan-by-scan basis for each TEB detector and for each side of the scan mirror. The BB WUCD is used to characterize the instrument nonlinear response coefficients on-orbit. The linear coefficient of the response function for each TEB is calibrated scan-by-scan with the nonlinear coefficient and offset from a LUT [11, 12]. 
The TEB calibration is based on a quadratic algorithm that converts the digital response of the sensor to calibration radiance $\left(L_{C A L}\right)$. The calibration radiance from the $\mathrm{BB}$ is adjusted for instrument self-emission due to the response versus scan angle (RVS) effect,

$$
L_{C A L}=R V S_{B B} \varepsilon_{B B} L_{B B}+\left(R V S_{S V}-R V S_{B B}\right) L_{S M}+R V S_{B B}\left(1-\varepsilon_{B B}\right) \varepsilon_{c a v} L_{c a v}
$$

where $\varepsilon$ is the emissivity of the sources and $L$ is the radiance from $\mathrm{BB}$, scan mirror, and cavity. A quadratic function is used for the calibration of the instrument response, as shown in Eq. (2)

$$
L_{C A L}=a_{0}+b_{1} d n_{B B}+a_{2} d n_{B B}^{2}
$$

Equations (1) and (2) are used for the WUCD calibration, with the offset term $\mathrm{a}_{0}$, the quadratic term $\mathrm{a}_{2}$ and digital response of the $\mathrm{BB}$ given in digital count $\left(\mathrm{dn}_{\mathrm{BB}}\right)$. The linear coefficient for Earth radiance retrieval can be expressed as

$$
b_{1}=\left[R V S_{B B} \varepsilon_{B B} L_{B B}+\left(R V S_{S V}-R V S_{B B}\right) L_{S M}+R V S_{B B}\left(1-\varepsilon_{B B}\right) \varepsilon_{c a v} L_{C A L}-a_{0}-a_{2} d n_{B B}^{2}\right] / d n_{B B}
$$

With the calibration coefficients, retrieval of Earth view radiance can be found using

$$
L_{E V}=\frac{1}{R V S_{E V}}\left[a_{0}+b_{1} d n_{E V}+a_{2} d n_{E V}^{2}-\left(R V S_{S V}-R V S_{E V}\right) L_{S M}\right]
$$

In the above calibration equations, the terms RVS and $\varepsilon$, are determined pre-launch whereas the $\mathrm{a}_{0}, \mathrm{a}_{2}$, and the band $21 b_{1}$ coefficients are updated quarterly (if necessary) using on-board BB WUCD measurements. Equations (1) and (2) summarize the calibration operation performed on all TEB detectors on a scan-by-scan basis. The calibration terms $\mathrm{a}_{0}$ and $\mathrm{a}_{2}$ shown in Equation (2) are computed on a quarterly basis during the $\mathrm{BB}$ WUCD process and after the cross-talk correction for the PV-LWIR bands.

The strategy for these updates in Terra C6.1 are summarized in Table 1. In addition, band $21 \mathrm{~b}_{1}$ is derived using BB cool down events with the offset and nonlinear term forced to zero in the fitting (not shown in Table 1). For C6.1, the BB dn is corrected for PV LWIR bands 27-30 cross-talk.

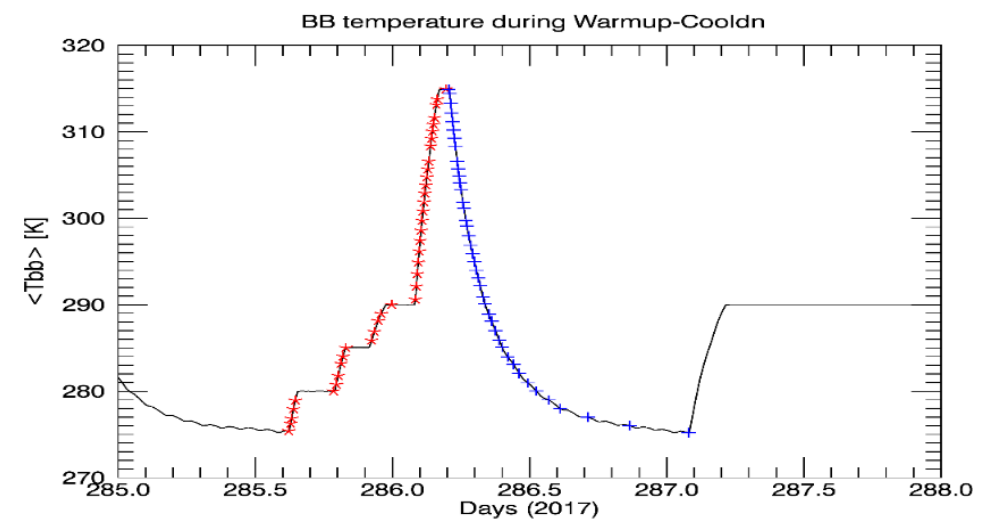

Figure 1. The BB temperature during an on-orbit WUCD event. The red symbols are the WU data, with the $\mathrm{CD}$ data in blue used for calibrations. For C6.1, CD data are used for deriving the coefficients. 
Table 1. Terra MODIS C6.1 calibration algorithm and procedure for LUT updates.

\begin{tabular}{|c|c|c|c|c|c|c|}
\hline Band & $\begin{array}{l}\text { Center } \\
\text { wavelength } \\
(\mathrm{nm})\end{array}$ & $\begin{array}{c}\text { Typical } \\
\text { tomperature } \\
\text { (Ttyp) }\end{array}$ & $\begin{array}{l}\text { NEdT } \\
\text { (K) }\end{array}$ & $\begin{array}{l}\text { C6.1 callbration } \\
\text { algorlthm }\end{array}$ & $\begin{array}{l}\text { Cross-talk } \\
\text { correction }\end{array}$ & $\begin{array}{l}\text { LUT update } \\
\text { procedure }\end{array}$ \\
\hline 20 & 3788.3 & 300 & 0.05 & \multirow{9}{*}{ 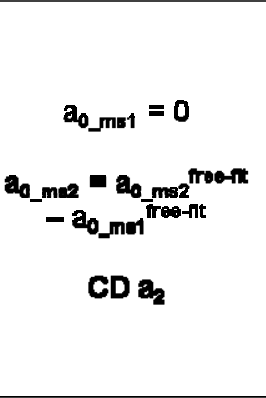 } & & \multirow{15}{*}{$\begin{array}{l}\text { If the Impacts on } \\
\text { L1B BT exceed the } \\
\text { criterlon (2*NEdT@ } \\
\text { Ltyp and } 2^{*} \mathrm{NEdT} \\
0.3 \text { Ltyp), an update } \\
\text { is issued. } \\
\text { The update uses a } \\
\text { moving window } \\
\text { average of last four } \\
\text { measurements of a0 } \\
\text { and a2. }\end{array}$} \\
\hline 22 & 3972.0 & 300 & 0.07 & & & \\
\hline 23 & 4056.7 & 300 & 0.07 & & & \\
\hline 24 & 4473.2 & 250 & 0.25 & & & \\
\hline 25 & 4545.4 & 275 & 0.25 & & & \\
\hline 27 & 6770.6 & 240 & 0.26 & & & \\
\hline 28 & 7342.9 & 250 & 0.25 & & $\begin{array}{c}\text { PV } \\
\text { electronic }\end{array}$ & \\
\hline 29 & 8628.7 & 300 & 0.08 & & crose-talk & \\
\hline 30 & 9734.1 & 250 & 0.25 & & & \\
\hline 31 & 11018.9 & 300 & 0.08 & \multirow{6}{*}{$\begin{array}{l}a_{0}=0 \\
C D a_{2}\end{array}$} & & \\
\hline 32 & 12032.1 & 300 & 0.05 & & \multirow{5}{*}{$\begin{array}{c}P C \\
\text { optical } \\
\text { cross-talk }\end{array}$} & \\
\hline 33 & 13366.0 & 260 & 0.26 & & & \\
\hline 34 & 13683.3 & 250 & 0.25 & & & \\
\hline 38 & 13913.2 & 240 & 0.28 & & & \\
\hline 36 & 14195.6 & 220 & 0.35 & & & \\
\hline
\end{tabular}

\subsection{Terra safe mode}

The Terra spacecraft entered safe mode in February of 2016 (02/18/2016: 14:33:17Z) during an inclination adjust maneuver. This caused MODIS to enter safe mode as well in which the nadir and space view doors were closed. On 02/22/2016, the Terra spacecraft was successfully recovered from safe mode back to science mode, with the key telemetry temperatures, such as BB, instrument temperature, electronic temperature, and CFPA temperature, stabilized after day 02/27/2016. A gain change was observed for most detectors along with increased noise levels. Figure 2 shows the response changes of selected TEB where band 31 shows stable behavior and bands 27 and 30 have large cross-talk impacts. A WUCD calibration was performed from $03 / 02 / 2016$ to $03 / 05 / 2016$. With updated QA and calibration coefficients $\left(\mathrm{a}_{0}, \mathrm{a}_{2}\right)$, the LUT was delivered on 03/10/2016 (2016/070). The 2016 safe mode caused a large impact on response and stability, including calibration coefficients and noise equivalent temperature difference (NEdT), on PV LWIR bands 27-30. The magnitude of electronic crosstalk contaminations for these bands was also significantly affected [10]. 


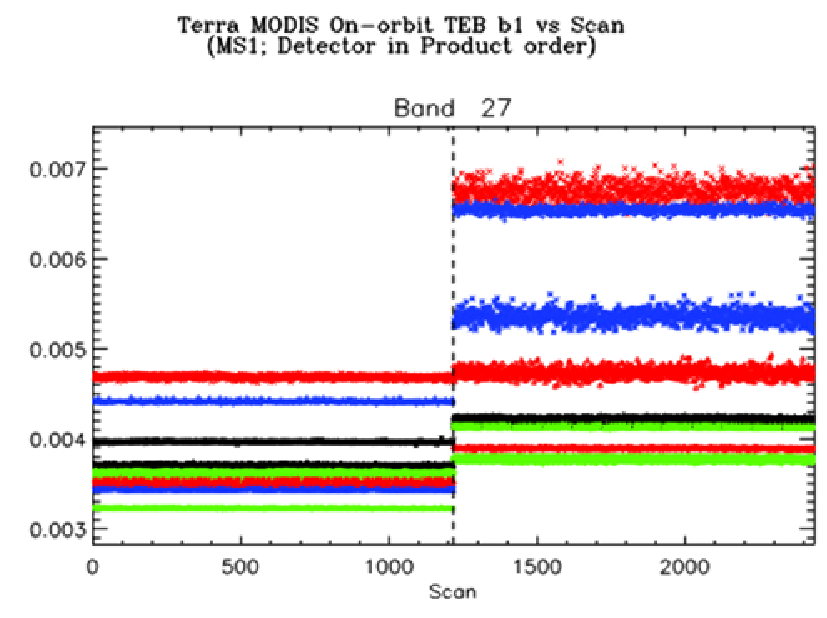

Terra MODIS On-orbit TEB b1 vs Scan

(MS1; Detector in Product order)

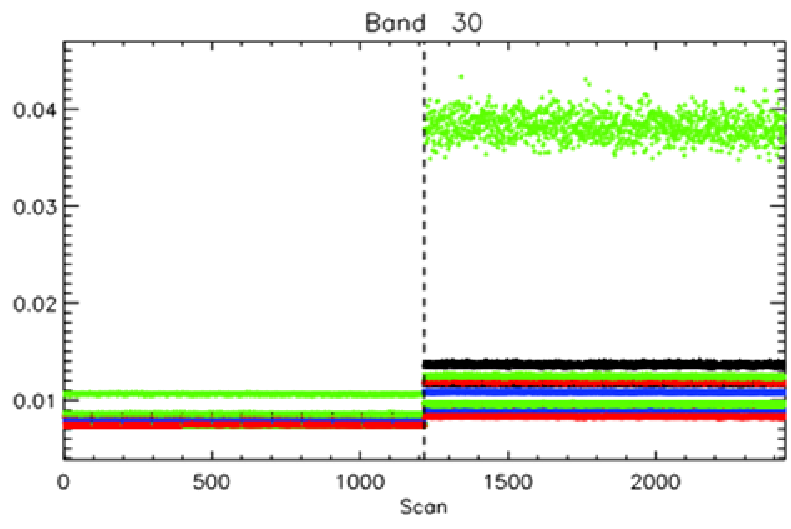

Terra MODIS On-orbit TEB b1 vs Scan (MS1; Detector in Product order)

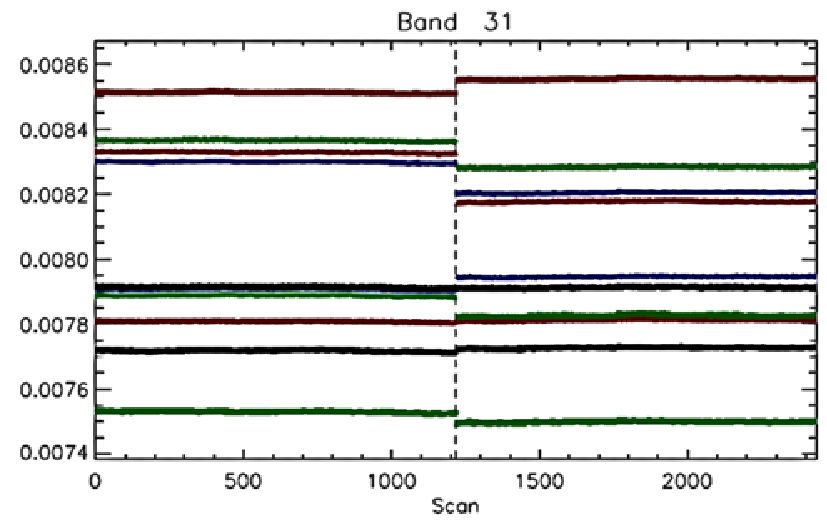

Figure 2. The instrument response changes due to safe mode for each detector of band 27 (top), 30 (middle), and 31 (bottom). The dashed line indicates the safe mode. 


\section{TERRA TEB CALIBRATION UPDATE}

The long-term trending of calibration coefficients shows the instrument stability and impact of instrument events, such as the safe mode in February 2016. Figures 3 and 4 show the long-term trending of bandaveraged calibration coefficients $\mathrm{a}_{0}$ and $\mathrm{a}_{2}$ of bands 27 to 30 , respectively. The $\mathrm{a}_{0}$ coefficients for mirror side 2 (mirror side $1 \mathrm{a}_{0}$ is set to 0 ) are relatively stable before safe mode and the updated coefficients are from the average of 4 WUCD events. After safe mode, because the instrument behavior showed significant changes, the $\mathrm{a}_{0}$ coefficient updates were derived from each individual WUCD event rather than from a moving average of the previous 4 WUCD events, with the $\mathrm{a}_{0}$ coefficient showing a jump for these 4 bands. Similarly, the $\mathrm{a}_{2}$ coefficients show an upward trend before the safe mode event. After recovery from safe mode, the first WUCD calibration was performed on 2016/062. These coefficient jumps might cause an impact on the L1B product. In addition, the algorithm of $\mathrm{a}_{0}$ coefficient is different for the two mirror side. The moving average procedure was reestablished after 4 WUCD events (approximately one year after safe mode) once the coefficients began trending nominally. The use of cold targets can be very helpful for the assessment of the $\mathrm{a}_{0}$ uncertainty impact on the L1B product before and after this event. The warm secen should be used for assessment of $a_{2}$ uncertainty impact. From the feedback from science team and our analysis, the $a_{0}$ uncertainty has large impact.
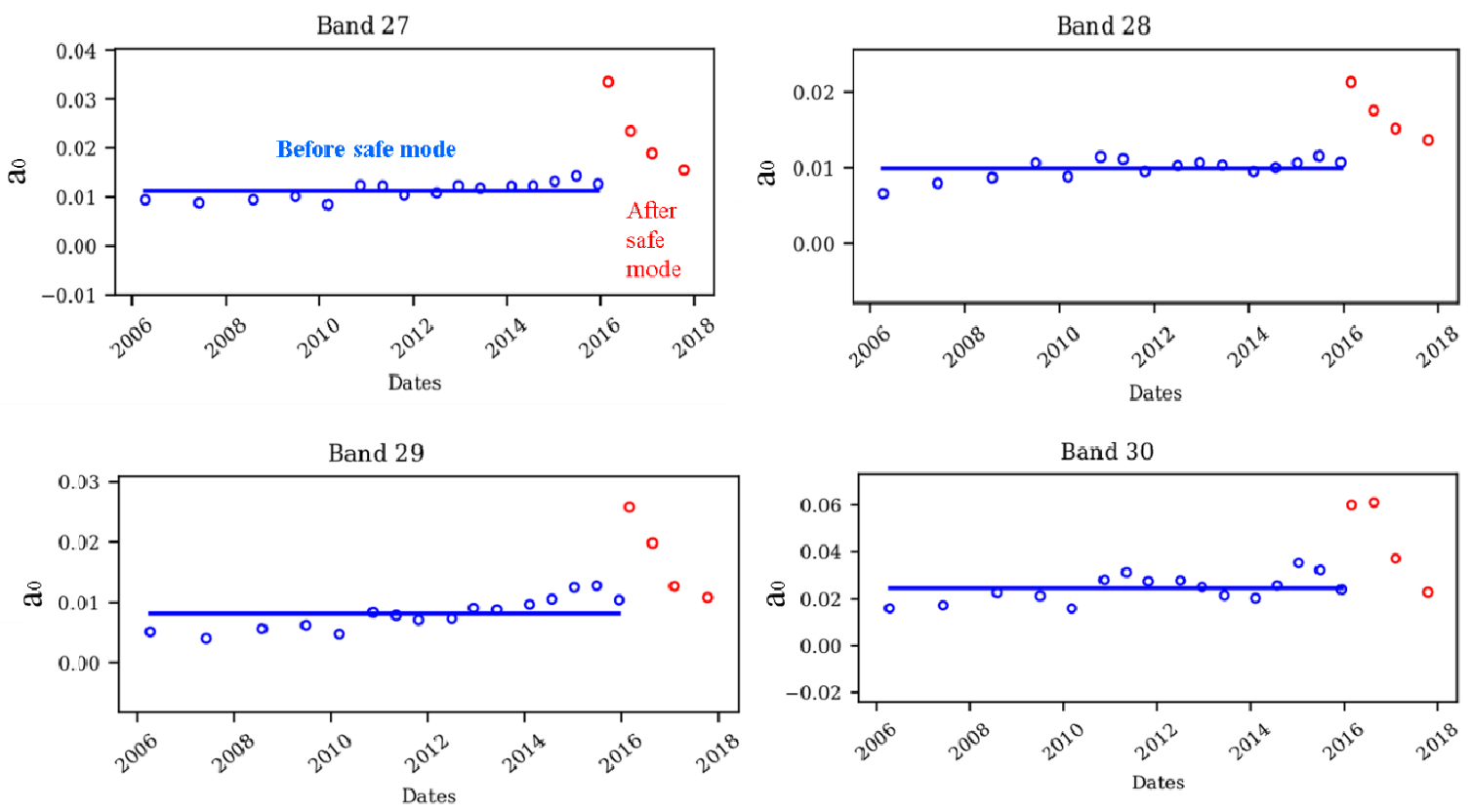

Figure 3. The long-term trending of band-averaged calibration coefficients $\mathrm{a}_{0}$ for mirror side 2 (mirror side 1 $\mathrm{a}_{0}$ is set to 0 ) of delivered LUTs for bands 27 to 30 . The line is the average of the coefficients before safe mode. 

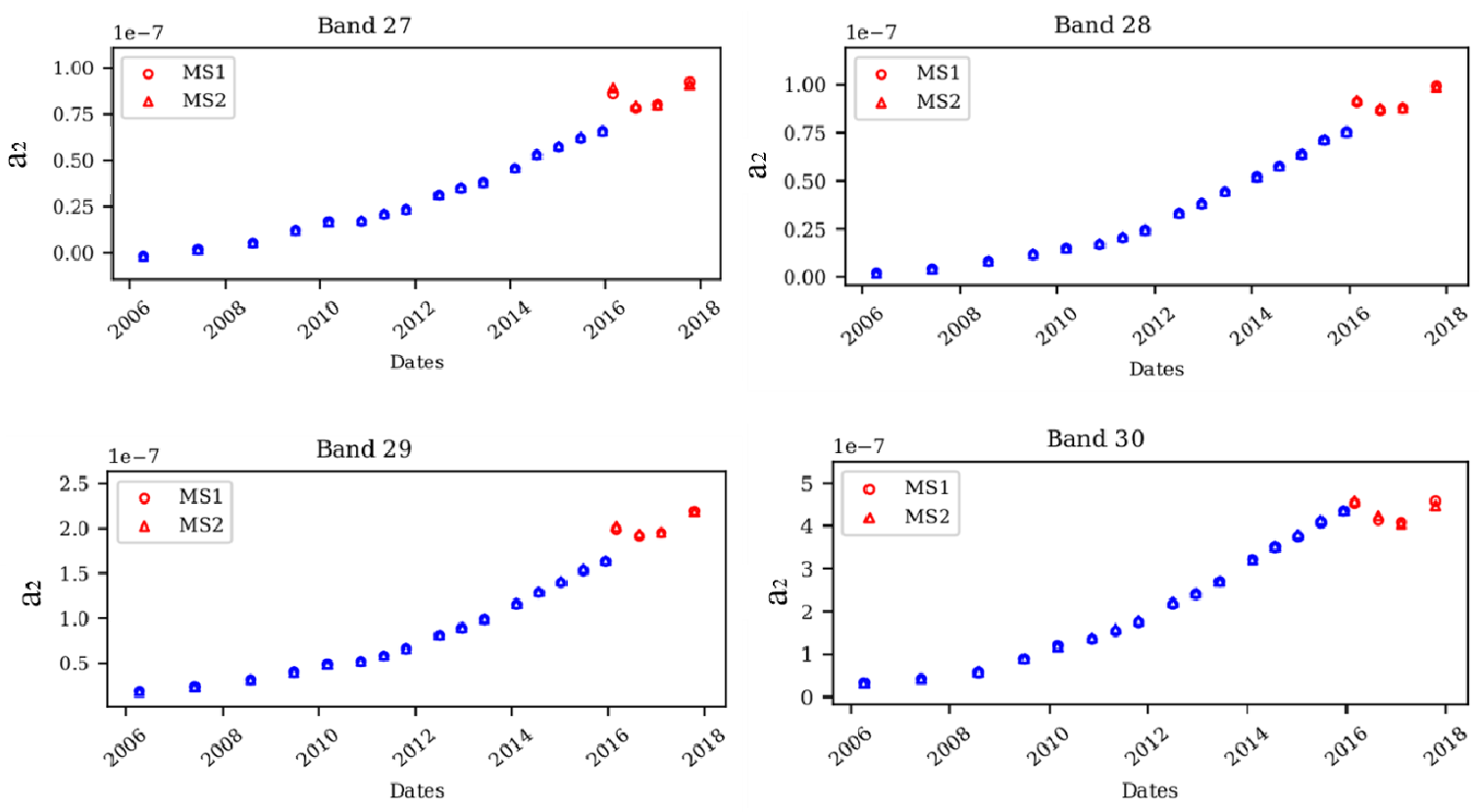

Figure 4. The long-term trending of band-averaged calibration coefficients $\mathrm{a}_{2}$ of delivered LUTs for bands 27 to 30 . The $\mathrm{a}_{2}$ for the two mirror sides are very close.

\section{CALIBRATION CONSISTENCE ASSESSMENT}

\subsection{Assessment using Dome-C}

\subsubsection{Dome-C BT measurement}

Dome-C $\left(75.12^{\circ} \mathrm{S}, 123.395^{\circ} \mathrm{E}\right)$ in Antarctica, as shown in Figure 5, has been identified as one of ground targets for evaluating and validating the sensors calibration accuracy. It is one of the most homogeneous Earth View (EV) targets and is situated near the Italian-French base of Concordia in a high polar plateau region with a mean elevation of $3200 \mathrm{~m}$. The typical seasonal temperature variation ranges from $200 \mathrm{~K}$ in winter to $250 \mathrm{~K}$ in summer. Due to relative uniformity, long-term stability, dry atmosphere, low aerosol loading and wind speed, low cloud cover and frequent satellite overpasses, Dome-C is well-suited for tracking the calibration stability and consistency of satellite sensors. It has been identified as one of ground targets for evaluating and validating the calibration accuracy of different sensors by the Committee on Earth Observation Satellites-Working Group on Calibration and Validation (CEOS-WGCV) [13-15]. Since 1995, the University of Wisconsin-Madison's Antarctic Meteorological Research Center (AMRC) has operated an Automatic Weather Station (AWS) at Dome-C collecting various meteorological measurements at 10-minute intervals and the data is available through the File Transfer Protocol site (//amrc.ssec.wisc.edu/pub/aws/). Because Dome-C is a high-latitude site, Terra passes over the site multiple times per day. In this work, only near-nadir MODIS observations of Dome-C are used in order to eliminate any possible bias due to scan angle affects. 

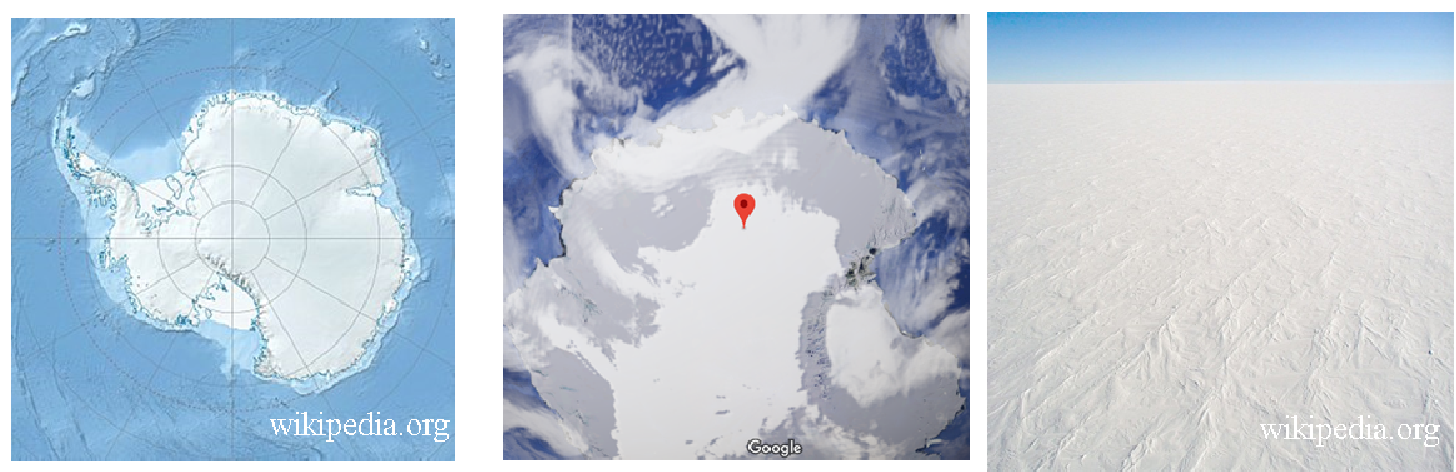

Figure 5. Illustration of Dome-C site used for sensor performance trending and comparison.

\subsubsection{Results}

In this paper, the assessment focuses on the mirror side differences at low BT. The mirror side difference is defined as mirror side 2 measurement minus mirror side 1 measurement. Typical Dome-C temperature ranges from $200 \mathrm{~K}$ to $250 \mathrm{~K}$ and provides feasibility to assess the impact of the $\mathrm{a}_{0}$ uncertainty on the L1B products at low temperatures. Figure 6 shows the mirror side difference for bands 27 to 30 from January of 2015 to January 2018, with the red vertical dashed line indicating the safe mode event and the purple lines are the dates of the calibration coefficients $\mathrm{a}_{0}$ and $\mathrm{a}_{2}$ LUT deliveries. The safe mode event causes a discontinuity in the trend of mirror side differences for these bands, which is likely due to the $\mathrm{a}_{0}$ LUT update. After safe mode, the mirror side differences gradually return to pre-safe mode levels. This assessment shows the impacts of safe mode along with the differences between the $\mathrm{a}_{0}$ calibration algorithms of the two mirror sides. 

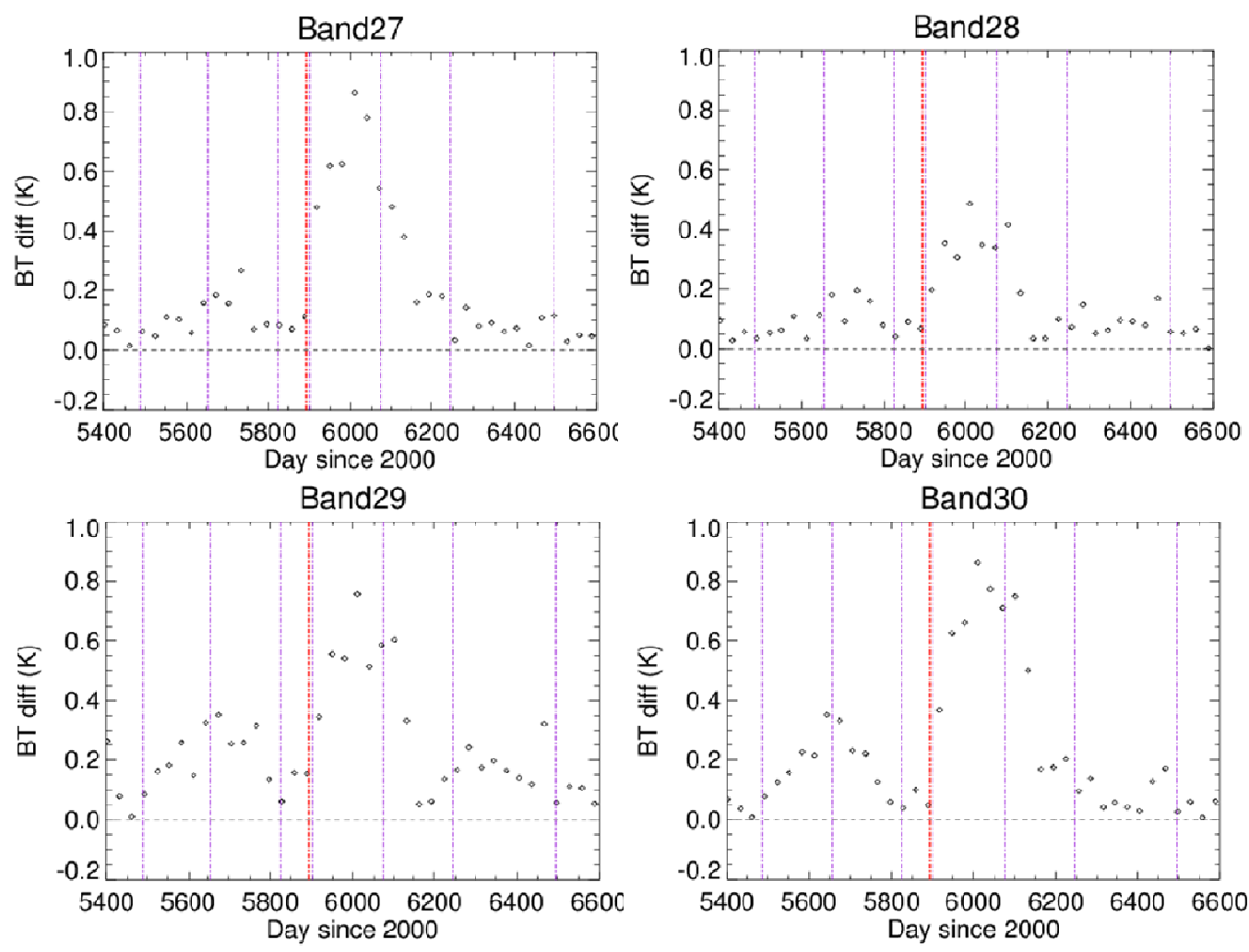

Figure 6. The mirror side difference for bands 27 to 30 from 2015 to January 2018 from the Dome-C site. The red vertical dashed line indicates the 2016 safe mode and the purple lines are the dates of the calibration coefficients $\mathrm{a}_{0}$ and $\mathrm{a}_{2}$ LUT deliveries.

\subsection{Assessment using DCC}

\subsubsection{DCC characterization}

DCCs are cold bright targets that are near-Lambertian at low solar and view zenith angles. Their consistent reflectance in the visible and near infrared (VIS/NIR) spectrum, combined with a minimal amount of water vapor and aerosols when viewed from space, makes them suitable targets for sensor calibration and intercomparison for the reflective bands of satellite sensors [16, 17]. Doelling et.al using a DCC technique to verify instrument calibration and instrument gain degradation [16]. DCC techniques have also been applied to the calibration of geostationary (GEO) sensors that typically have limited on-orbit calibration capability. Most of the work associated with DCCs is currently focused on sensor inter-comparison and stability assessment of reflective bands. DCCs are also used for MODIS performance assessments [18, 19].

The homogeneity of the visible reflectance and IR BT can be used to remove pixels at the cloud edge. The DCC pixel identification and selection in this work followed the procedure described in reference $[16,18]$. Potential DCC pixels are identified as any pixel with a 11- $\mu \mathrm{m}$ (MODIS band 31) BT less than $205 \mathrm{~K}$ within the latitude range of $30^{\circ} \mathrm{N}$ to $30^{\circ} \mathrm{S}$ over all surface types. The standard deviations of the IR temperature and the visible $0.6 \mu \mathrm{m}$ reflectance were computed over all $3 \times 3$ pixel blocks surrounding each potential DCC pixel. If the standard deviation of the IR temperature was larger than the threshold of $1 \mathrm{~K}$ or the reflectance 
standard deviation was larger than 3\%, this potential DCC pixel was discarded to remove the DCC pixels from the cloud edges. Figure 7 shows a DCC image from MODIS observations.

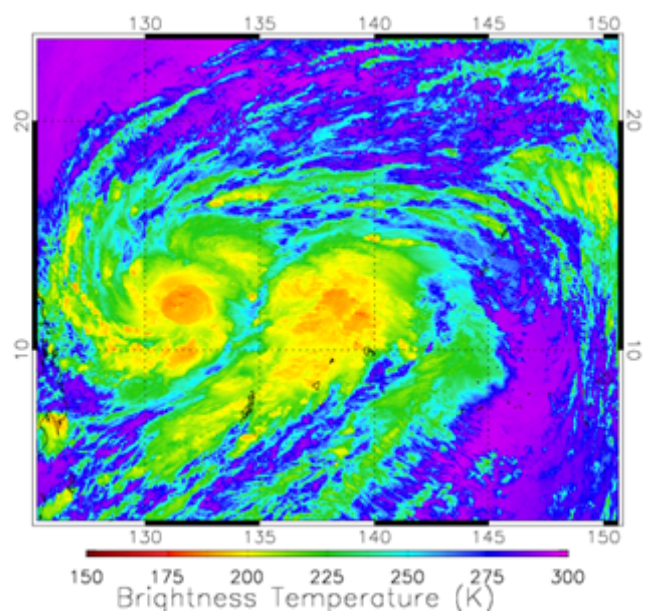

Figure 7. An example of a DCC image from MODIS observations (granule 2014305.0125).

\subsubsection{Results}

The BT of DCCs for MODIS band 31 should be below $205 \mathrm{~K}$. Figure 8 shows the comparison of the temperature distribution between the two mirror sides before and after safe mode, with the top two plots showing band 31 and the bottom plots showing band 27. The distribution is defined as the histogram normalized to its maximum. For band 31 there is no significant mirror side difference and no significant safe mode impact. For band 27, however, there is a slight mirror side difference before safe mode. This mirror side difference in band 27 becomes enlarged after the safe mode event. Figure 9 shows the mirror side difference for bands 27 to 30 from 2015 to January 2018, derived from DCC observations, with the red vertical dashed lines indicating the safe mode event and purple lines are the dates of the calibration coefficients $\mathrm{a}_{0}$ and $\mathrm{a}_{2}$ LUT deliveries. The safe mode event causes a jump in the mirror side difference which is generally due to the $\mathrm{a}_{0}$ LUT change. Similar to the Dome-C results, the mirror side difference gradually returns back to pre-safe mode levels. This assessment also shows the impacts of safe mode and difference of $\mathrm{a}_{0}$ calibration algorithm of the two mirror sides. 

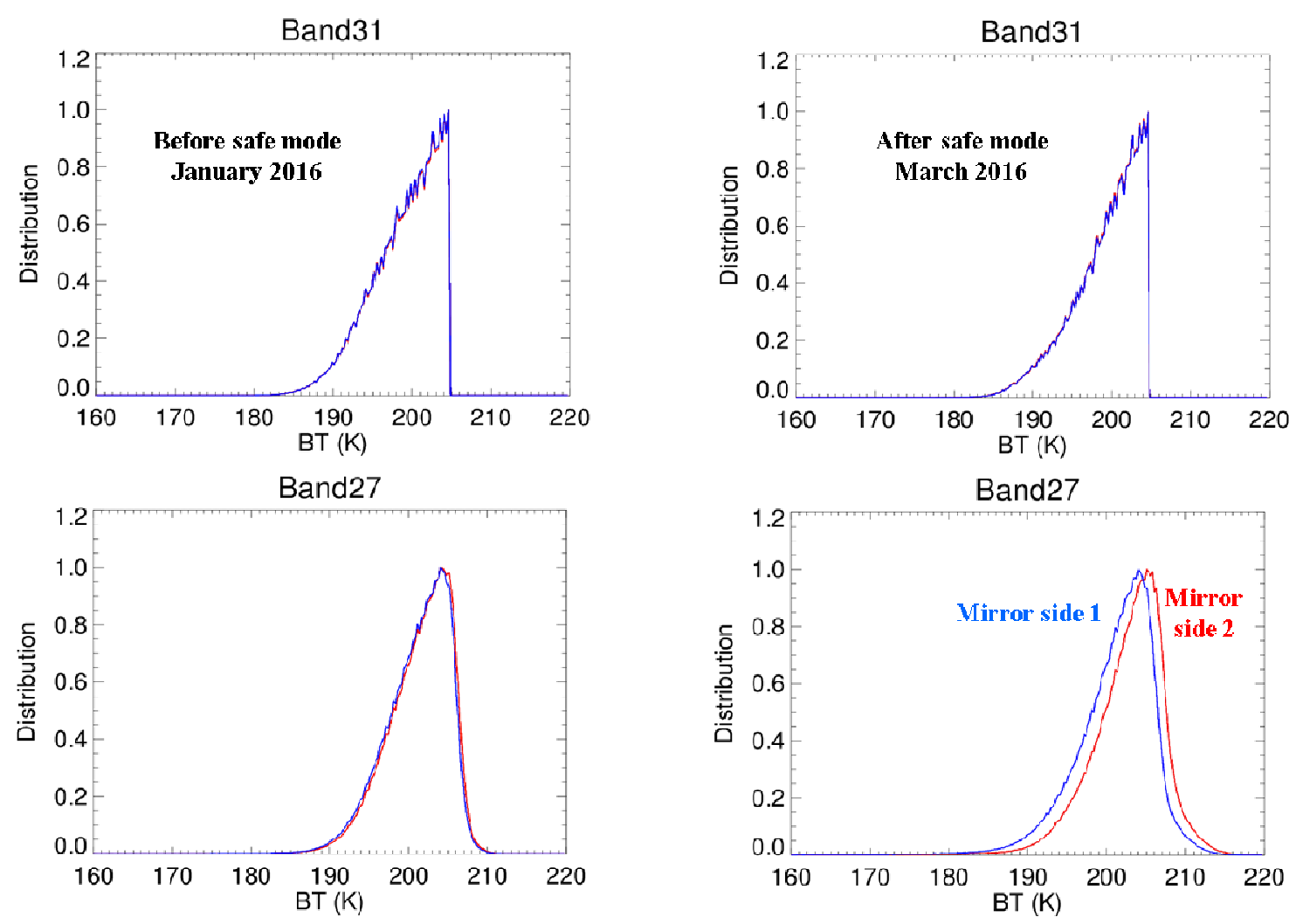

Figure 8 . The comparison of the temperature distribution between the two mirror sides before and after safe mode. The top two plots are for band 31 and bottom plots for band 27. Left side plots are before safe mode while right plots are for after safe mode. Blue is for mirror side 1 and red is for mirror side 2. 

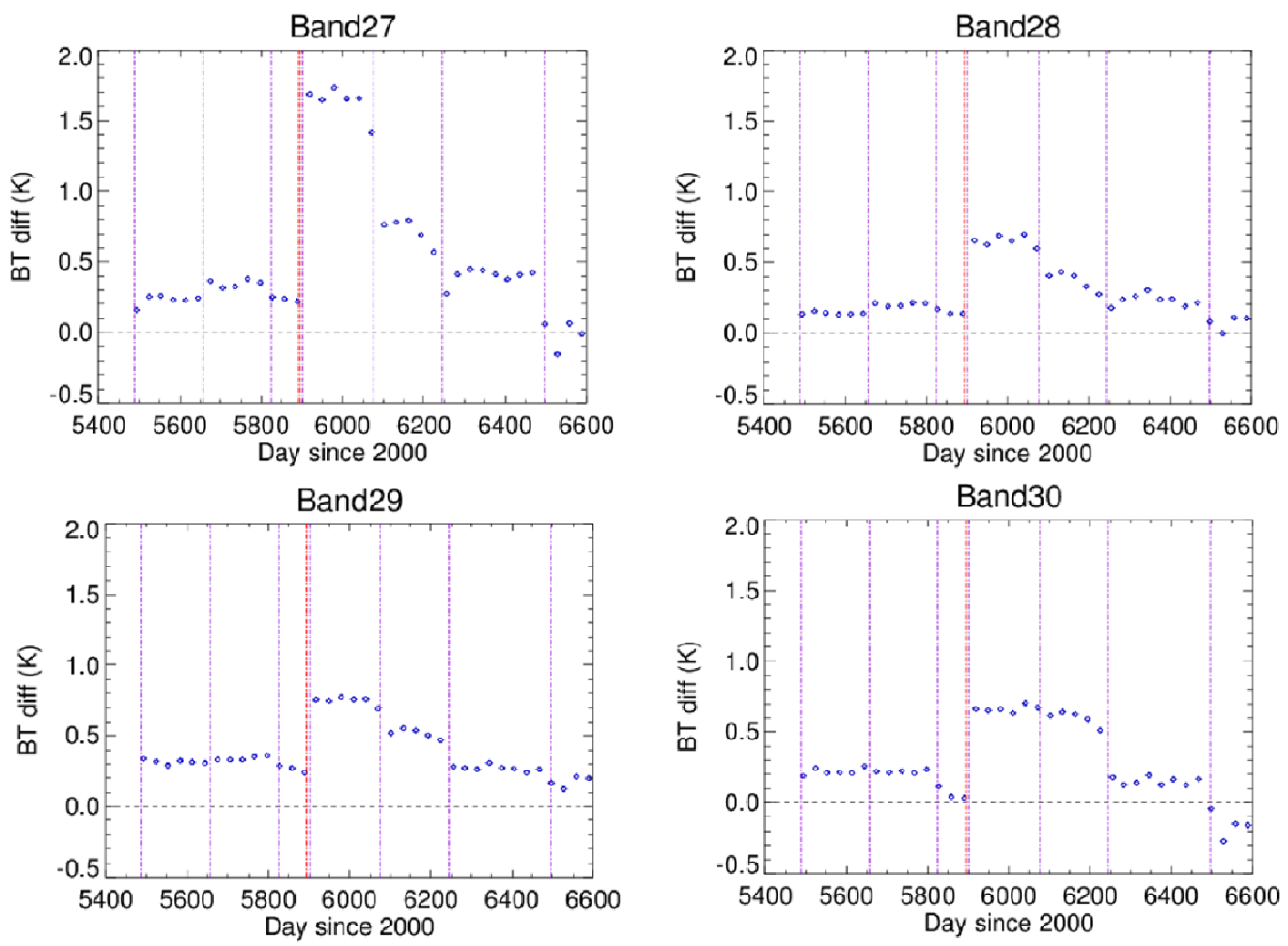

Figure 9. The mirror side difference derived from DCC observations for bands 27 to 30 from 2015 to January 2018. The red vertical dashed line indicates the safe mode and purple lines are the dates of the calibration coefficients $\mathrm{a}_{0}$ and $\mathrm{a}_{2}$ LUT deliveries.

\subsection{Assessment using cavity temperature of scheduled lunar event}

\subsubsection{Scheduled lunar event}

MODIS lunar observations have been extensively used to support the on-orbit calibration and characterization, including radiometric stability monitoring for RSB and TEB, spatial characterization for sensor band to band registration (BBR) and modulation transfer function (MTF), optical leak and crosstalk, and sensor calibration inter-comparison [20,21]. MODIS lunar observations are scheduled approximately monthly with the lunar phase angle within a small range of $55^{\circ}$ to $56^{\circ}$ for Terra MODIS, with the waning moon viewed through the SV port. The scheduled lunar observations are made during the spacecraft nighttime using spacecraft roll maneuvers. A data sector rotation is applied to capture the moon image at the Earth view sector. In this work, to assess the calibration impact on cold targets, the cavity temperature measurements are used rather than the moon image. Figure 10 shows the MODIS cavity and the image during the scheduled moon events. 


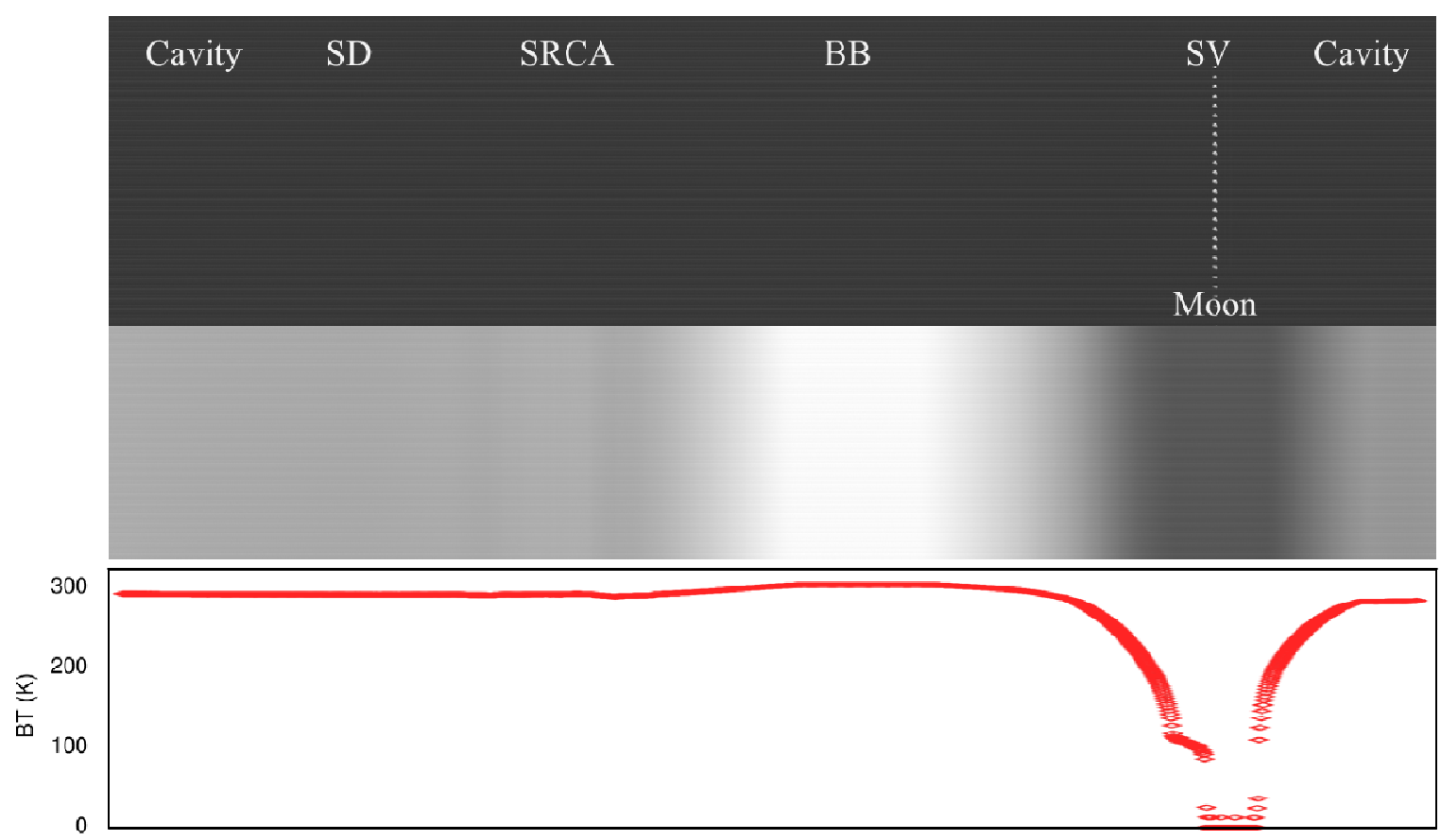

Figure 10. The top image during a lunar event with data sector rotation. The moon is shown in the frames corresponding to the SV. The lower image shows the cavity BT of band 31 after the lunar observation.

\subsubsection{Results}

The selection of the portion of the cavity during lunar events uses band 31 BT around the telemetry cavity temperature. The selected frame are used for all the bands to assess the calibration consistency of the mirror side differences. The space view is used as background. Figure 11 shows the BT mirror side differences for bands 27 to 30 from 2015 to January 2018 with focus on the safe mode impact. The red vertical dashed line indicates the safe mode event and purple lines are the dates of the calibration coefficients $a_{0}$ and $a_{2}$ LUT deliveries. The safe mode event causes a jump in the mirror side difference which is generally due to the $\mathrm{a}_{0}$ LUT change. These results are consistent with Dome-C and DCC results. After the jump caused by safe mode, the mirror side gradually returns to pre-safe mode levels. This assessment also shows the impacts of safe mode and difference in the $\mathrm{a}_{0}$ calibration algorithm of the two mirror sides. 

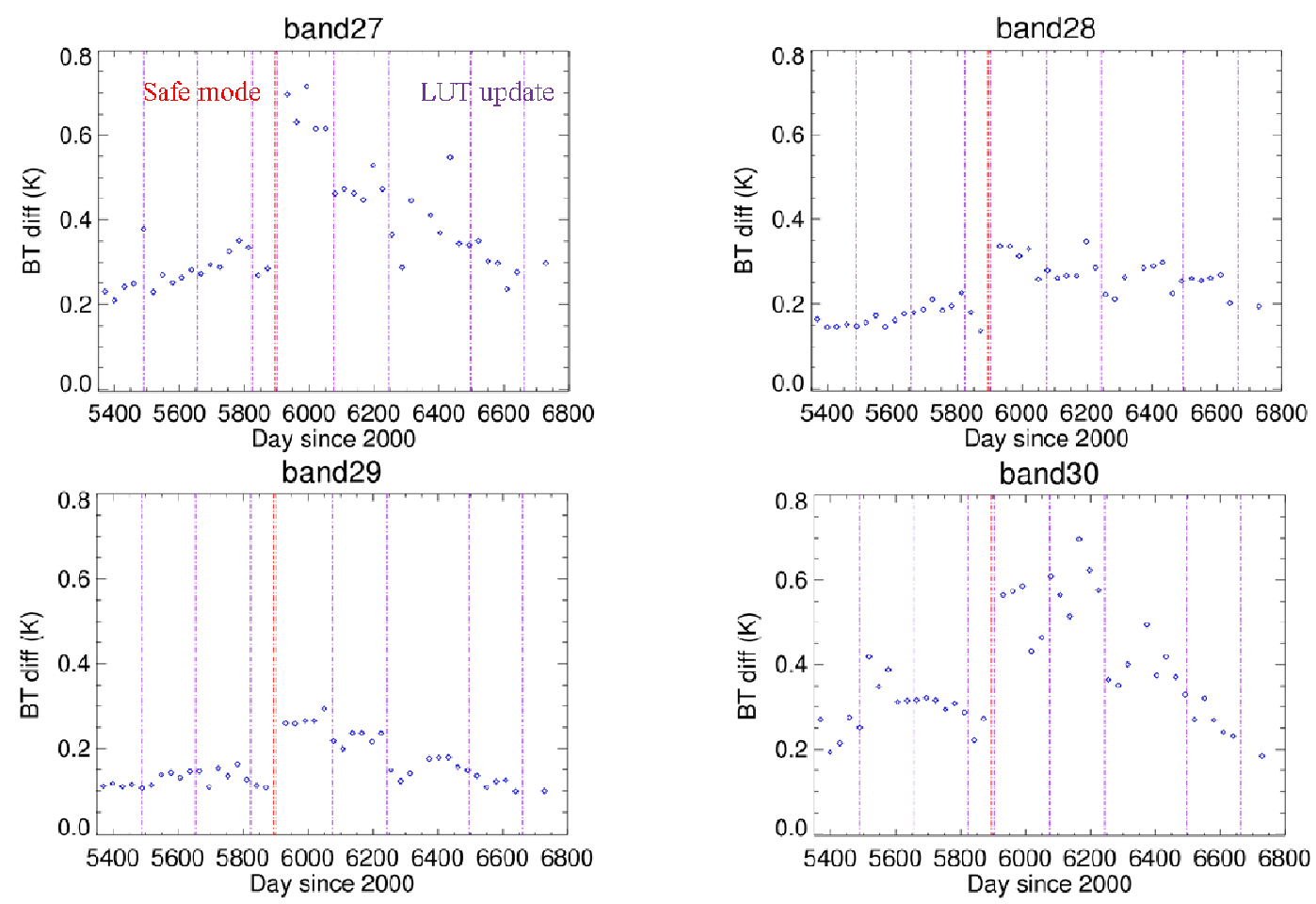

Figure 11. The BT mirror side differences for bands 27 to 30 from 2015 to January 2018 using the cavity measurements during lunar events. The red vertical dashed line indicates the safe mode event and purple lines are the dates of the calibration coefficients $\mathrm{a}_{0}$ and $\mathrm{a}_{2}$ LUT deliveries.

\section{SUMMARY}

The crosstalk effects on the Terra MODIS PV LWIR bands 27 to 30 have increased significantly in recent years, especially after the safe mode in February 2016. After this event, the instrument response initially changed rapidly and the $\mathrm{a}_{0}$ coefficient update derived from each individual WUCD event rather than the presafe mode 4 WUCD average. The moving average procedure was reestablished after 4 WUCD events (approximately one year after safe mode), with the $\mathrm{a}_{0}$ uncertainty having impacted the L1B product. In Collection 6.1, the PV LWIR crosstalk is characterized and corrected using lunar measurements. Accurate determination and update of the offset coefficient using measurements from BB WUCD are challenging. This work focuses on the assessment of calibration consistency and the delivery procedure impact on the L1B product.

The use of observations over cold targets provide feasibility of analyzing the offset impact on the products. The cold targets, Dome-C, DCCs, and cavity measurement during scheduled lunar events are used in this work. Due to the atmospheric effects, the results from Earth targets may have extra bias. The extra bias can be also scene dependent and seasonal dependent. For the impact from uncertainty of $\mathrm{a}_{0}$, the impact on L1B should be BT dependent. The Dome-C temperature has seasonal variation and range from $200 \mathrm{~K}$ to $250 \mathrm{~K}$. This variation can cause changes of the mirror side difference for the same $\mathrm{a}_{0}$ error. DCC temperature is more stable since the pixel selection uses band $31 \mathrm{BT}$. The atmospheric effect on DCC is lower. The results from DCC is more stable. The cavity BT is stable and there is no any atmospheric effect. The result should be more reliable than other Earth view measurements around the same BT range. 


\section{REFERENCES}

[1] W. L. Barnes and V. V. Salomonson, "MODIS: A global image spectroradiometer for the Earth Observing System,” Crit. Rev. Opt. Sci. Technol., vol. CR47, 285-307, 1993.

[2] W. L. Barnes, V. V. Salomonson, B. Guenther, and X. Xiong, "Development, characterization, and performance of the EOS MODIS sensors," in Proc. SPIE, 5151, 337-345, 2003.

[3] W. L. Barnes, X. Xiong, and V. V. Salomonson, "Status of Terra MODIS and Aqua MODIS," J. Adv. Space Res., 32(11), 2099-2106, 2003.

[4] V. V. Salomonson, W. L. Barnes, X. Xiong, S. Kempler, and E. Masuoka, "An overview of the Earth Observing System MODIS instrument and associated data systems performance," in Proc. IEEE IGARSS, 1174-1176, 2002.

[5] C. L. Parkinson, "Summarizing the first ten years of NASA's Aqua mission", IEEE J. Sel. Topics Appl. Earth Observ. Remote Sens., 6(3), 1179-1188, 2013.

[6] Yue, H., C. He, Y. Zhao, Q. Ma; Q. Zhang, "The brightness temperature adjusted dust index: An improved approach to detect dust storms using MODIS imagery". INTERNATIONAL JOURNAL OF APPLIED EARTH OBSERVATION AND GEOINFORMATION, 57, 166-176, 2017.

[7] Skakun, S., CO Justice, E Vermote, JC Roger Transitioning from MODIS to VIIRS: an analysis of interconsistency of NDVI data sets for agricultural monitoring" International journal of remote sensing, 39(4), 971-992, 2018

[8] Gupta, Pawan; Remer, Lorraine A; Levy, Robert C; Mattoo, Shana, "Validation of MODIS 3 km land aerosol optical depth from NASA's EOS Terra and Aqua missions" Atmospheric Measurement Techniques; Katlenburg-Lindau 11 (5), 2018

[9] R. C. Levy, S. Mattoo, L. A. Munchak, L. A. Remer, A. M. Sayer, F. Patadia, and N. C. Hsu, "The Collection 6 MODIS aerosol products over land and ocean", Atmos. Meas. Tech., 6, 2989-3034, 2013

[10] Wilson, T., A. Wu, A.. Shrestha, X. Geng, Z. Wang, C. Moeller, R. Frey, and X. Xiong, "Development and Implementation of an Electronic Crosstalk Correction for Bands 27-30 in Terra MODIS Collection 6", Remote Sensing, vol. 9(6), issue 569, 2017

[11] Xiong, X., B. Wenny, A. Wu, and V. Salomonson, "Aqua MODIS Thermal Emissive Bands On-orbit Calibration, Characterization, and Performance", IEEE Trans. Geosci. Remote Sens., vol. 47, issue 3, pp. 803-814, 2009

[12] Xiong, X., A. Wu, B. N. Wenny, S. Madhavan, Z. Wang, Y. Li, N. Chen, W. Barnes, and V. Salomonson, "Terra and Aqua MODIS Thermal Emissive Bands On-Orbit Calibration and Performance", IEEE Transactions on Geoscience and Remote Sensing, vol. 53, issue 10, pp. 5709 - 5721, 2015.

[13] Xiong, X., A. Wu, and B. Wenny, "Using Dome C for MODIS Calibration Stability and Consistency", J. Appl. Remote Sens., vol. 3, no. 033520, 2009.

[14] Cao, C., S. Uprety, X. Xiong, A. Wu, P. Jing, D. Smith, G. Chander, N. Fox, and S. Ungar, "Establishing the Antarctic Dome C Community Reference Standard Site towards Consistent Measurements from Earth Observation Satellites", Canadian Journal of Remote Sensing, vol. 36, issue 5, 2010

[15] Wenny, B., and X. Xiong, "Using a Cold Earth Surface Target to Characterize Long-term Stability of the MODIS Thermal Emissive Bands", IEEE Geosci. Remote Sens. Let., vol. 5, issue 2, pp. 162-165, 2008

[16] Doelling, D. R., L. Nguyen, P. Minnis (2004), On the use of deep convective clouds to calibrate AVHRR data, in Proc. SPIE, Earth Observing Systems IX, pp. 281-299

[17] Doelling, D.R., G. Hong, D. Morstad, R. Bhatt, A. Gopalan, and X. Xiong (2010), The Characterization of Deep Convective Cloud Albedo as a Calibration Target Using MODIS Reflectances, in Proc. SPIE, Earth Observing Missions and Sensors: Development, Implementation, and Characterization, vol. 7862, 78620I-1 to $78620 \mathrm{I}-10$ 
[18] Chang, T., X. Xiong, A. Angal, and Q. Mu, "Assessment of MODIS RSB detector uniformity using deep convective clouds", Journal of Geophysical Research: Atmospheres, vol. 121, issue 9, pp. 4783-4796, 2016

[19] Mu, Q., A. Wu, X. Xiong, D. R. Doelling, A. Angal, T. Chang, and R. Bhatt, "Optimization of a Deep Convective Cloud Technique in Evaluating the Long-Term Radiometric Stability of MODIS Reflective Solar Bands", Remote Sensing, vol. 9 (6), issue 535, 2017

[20] Barnes, W., X. Xiong, R. E. Eplee, Jr., J. Sun, and C. Lyu, "Use of the Moon for Calibration and Characterization of MODIS, SeaWiFS, and VIRS", Earth Science Satellite Remote Sensing, vol. 2, New York, Springer-Verlag, pp. 98-119, 2006.

[21] Xiong, X., J. Sun, S. Xiong, and W. Barnes, "Using the moon for MODIS on-orbit spatial characterization, Sensors, Systems, and Next-Generation Satellites VII", Proc. SPIE, vol. 5234, pp. 480487,2003 